\title{
Simulating Particle Swarm Optimization Algorithm to Estimate Likelihood Function of $\operatorname{ARMA}(1,1)$ Model
}

\author{
Basad Ali Hussain Al-sarray \\ Laboratoire de Mathematiques de Besancon Universite de Franche-Comte, Besancon cedex, France.
}

Received: May 12, 2015 / Accepted: June 09, 2015 / Published: October 25, 2015.

\begin{abstract}
This paper present a simulation study of an evolutionary algorithms, Particle Swarm Optimization PSO algorithm to optimize likelihood function of $\operatorname{ARMA}(1,1)$ model, where maximizing likelihood function is equivalent to maximizing its logarithm, so the objective function 'obj.fun' is maximizing log-likelihood function. Monte Carlo method adapted for implementing and designing the experiments of this simulation. This study including a comparison among three versions of $P S O$ algorithm "Constriction coefficient CCPSO, Inertia weight IWPSO, and Fully Informed FIPSO", the experiments designed by setting different values of model parameters $a_{1}, b_{1}$ sample size $n$, moreover the parameters of $P S O$ algorithms. MSE used as test statistic to measure the efficiency $P S O$ to estimate model. The results show the ability of $P S O$ to estimate $A R M A^{\prime}$ s parameters, and the minimum values of MSE getting for COPSO.
\end{abstract}

Key words: Particle Swarm Optimization algorithm, Likelihood function, $A R M A(1,1)$ Model

\section{Introduction}

A mathematical optimization problem is one in which some real-valued function is either maximized or minimized relative to a given set of feasible alternatives. Mathematical optimization is a selection of "best available" values of the objective function defined in its domain, this selection of a best value according to some criteria.

That is, we consider optimization problems having a mathematical representation, a real function $f: X \rightarrow Y$, is known as fitness or the objective function, defined on a $X \subseteq \mathcal{R}^{d}$ domain of the function, and it is the search space of $x \in \mathcal{R}^{n}$, each of $x_{i}, i=1, \cdots d$ defined in particular range $\left[x_{i b_{i}},{ }_{u b_{i}}\right]$, mathematical formulation of a minimization problem is

$$
\begin{gathered}
\min _{\vec{x}} f(\vec{x}) \\
\text { s.t } x_{l b_{i}} \leq x_{i} \leq x_{u b_{i}}, \text { for } i=1, \cdots, d
\end{gathered}
$$

Corresponding author: Basad Ali Hussain Al-sarray, Laboratoire de Mathematiques de Besancon Universite de Franche-Comte. E-mail: basad.al-sarray@univ-fcomte.fr.
Most of researches prefer to use heuristic algorithms to optimize the solution of the optimization problems because it is easy to implement it and programming, also the availability of there software, in other side, we can see the complexity of the functions that used to describe a systems and there is no analytically solution encouraged the researchers to adopt this type of algorithms. ARMA model is one of models that its complexity increase with increasing it's orders, the number coefficients in the model. Likelihood function used to estimate $A R M A$ 's parameters, in fact the complexity of solving likelihood function also effected by increasing the model's order, using likelihood function to estimate model's parameters requires a numerical methods like fixed point method, Newton Raphson method, using these method need to find derivative of the likelihood function, the looping of searching about the solution starting by an initial guess solution this value may be far away from the optimal solution, these methods poor of a global convergence properties, i.e., these methods are iterative methods and almost there solutions are locally 


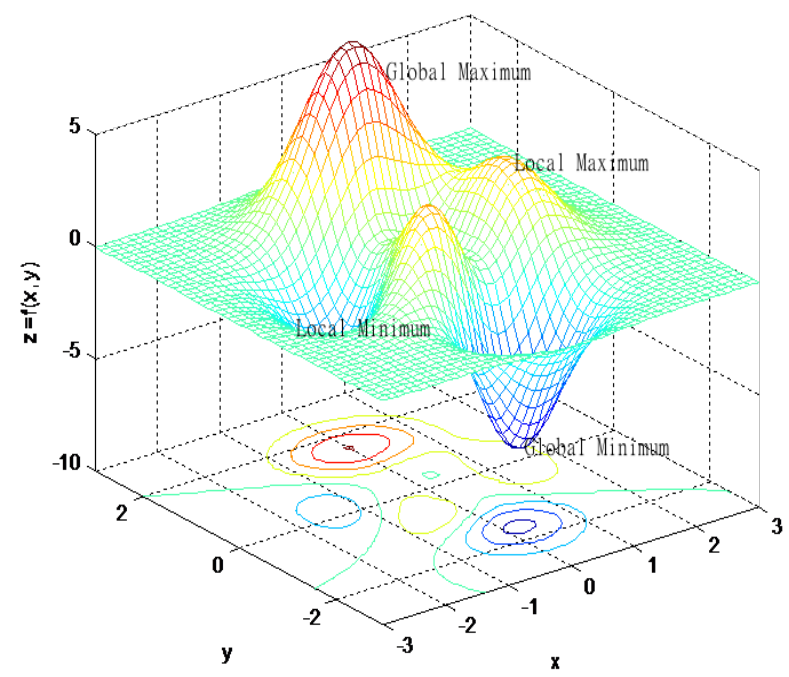

Fig. 1 An example of function with multi global and local maximum and minimum optimal point.

evaluated, and in case of zero computing derivative may loop indefinitely.

The purpose of this study is simulating one of the soft computing algorithms, known as Particle Swarm Optimization PSO algorithm [1], Eberhart and Kendy 1995, to optimize likelihood function of time series model autoregressive moving average $\operatorname{ARMA}(1,1)$ model.

The idea of PSO is an simulation to a natural behavior of flock of birds, fish.., during their searching about the food, it is known that the best way followed by flock to reach of an ideal sources of the food depend on the information keeping in their memory where each bird has particular memory about the best position, beside that, the connections and exchanging the information between each other support them to find the best position.

There are a lot of studies main in developing and using $P S O$ in time series analysis, it was used for model selection, identifying ARMAX models, chaotic time series modeling, model order reduction, and for prediction of chaotic system .... This study is a continuous to works given in [8],[9] and [10] that using different versions of soft computing algorithm for ARMA model estimation and forecasting, the aim of these studies is to show the ability of soft computing algorithms in time series modeling and its applications.

Rest of this paper given by, section 2 introduces ARMA model and likelihood function, section 3 present PSO and its versions, section 4 gives a simulation and the results, and the last section is for the conclusions.

\section{Autoregressiv Moving Average $\operatorname{ARM}(\mathrm{p}, \mathrm{p})$}

A statistical phenomenon that evolves in time according to probabilistic laws is called a stochastic process. In analysis time series we regarded it as a realization of a stochastic process. A very special case of stochastic process, called a stationary process, is based on the assumption that the process is in a particular state of statistical equilibrium. A stochastic process is said to be strictly stationary if it's properties are unaffected by change of time origin.

The special case of stationary process is an Autoregressive Integrated Moving Average ARIMA process, this process given by

$$
a(B) y_{t}=\Delta^{d} a(z) y_{t}=b_{0}+b(z) e_{t}
$$

where $\quad a(z)=1-a_{1} z+\cdots-a_{p} z^{p} \quad$ know as autoregressive operator, it is assumed to be stationary, the roots of $a(z)$ lie outside the unit circle.

$b(z)=1-b_{1} z-\cdots-b_{q} z^{q}$ is the moving average operator it is assumed to invertible the roots of $b(z)=0$ lie outside of unit circle, $z$ is here refer to the back-shift operator and $\Delta^{d}$ is difference operator, usually $d=1,2$ the difference take in order to make series more stable, $p, q$ is the order of ARMA.

The important representation of ARMA process this one given based on the previous noises where $y_{t}$ represented as a linear filter:

$$
\begin{gathered}
y_{t}=e_{t}+\psi_{1} e_{t-1}+\psi_{2} e_{t-2}+\ldots \\
=e_{t}+\sum_{j=1}^{\infty} \psi_{j} e_{t-j} \\
=\psi(z) e_{t}
\end{gathered}
$$


where $\psi(z)=1+\psi_{1} z+\psi_{2} z^{2}+\cdots$.

So, the stationary $\operatorname{ARMA}(p, q)$ process is given by

$$
y_{t}=\frac{b(z)}{a(z)}=\psi(z) e_{t}
$$

$\psi_{0}=1$ and $e_{t}$ is a sequence of uncorrelated shocks.

For this note we interest in special case of ARMA process, $\operatorname{ARMA}(1,1)$, this model given by,

$$
\left(1-a_{1}\right) y_{t}=\left(1-b_{1}\right) e_{t}
$$

as a result,

$$
y_{t}=a_{1} y_{t-1}+e_{t}-b_{1} e_{t-1}
$$

The linear filtering of this model is defined by finding $\psi$ 's as follows:

$$
\begin{gathered}
a(z) \psi(z)=b(z) \\
{\left[1-a_{1} z\right]\left[1+\psi_{1} z+\psi_{2} z^{2}+\cdots\right]=\left[1-b_{1} z\right]}
\end{gathered}
$$

by equaling powers of the coefficients for both sides

$$
\psi_{j}=a_{1}^{j-1}\left(a_{1}-b_{1}\right)
$$

and

$$
y_{t}=a_{1}^{j-1}\left(a_{1}-b_{1}\right) e_{t-j}+e_{t}
$$

\subsection{Likelihood Function of ARMA(1, 1)}

The likelihood function of $n$ random variables $x_{1}, x_{2}, \cdots, x_{n}$ is defined to be the joint density of the $n$ random variables, $\mathbf{P}_{x_{1}, x_{2}, \cdots x_{n}}\left(x_{1}, x_{2}, \cdots, x_{n} ; \theta\right)$, which is considered to be a function of $\theta$. In particular if $x_{1}, x_{2}, \cdots, x_{n}$ is a random sample from the density $\mathcal{P}(x ; \theta)$, then the likelihood function is $\mathbf{p}\left(x_{1}\right), \cdots, \mathbf{p}\left(x_{n}\right)$.

Be the likelihood function of the random variables $x_{1}, \cdots, x_{n}$. Assuming that $e_{t} \sim$ i.i.d.N $\left(0, \sigma_{e}^{2}\right)$, the joint probability density of $e=\left(e_{1}, e_{2}, \cdots, e_{n}\right)^{t}$ is given by

$$
\mathbf{P}(e)=\left(2 \pi \sigma_{e}^{2}\right)^{\frac{-n}{2}} \exp \left(-\frac{1}{2 \sigma_{e}^{2}} \sum_{t=1}^{n} e_{t}^{2}\right)
$$

Let $y_{t}, t=1,2, \ldots n$, be sequence of time series observations generated by stationary $\operatorname{ARMA}(1,1)$ process, with sample size $n$, the noise term in Eq. (4) can be written as:

$$
\mathbf{e}_{t}=y_{t}-a_{1} y_{t-1}+b_{1} e_{t-1}
$$

For a given initial values $\left(y_{*}, e_{*}\right)$, let

$$
\mathbf{L}=\mathbf{L}\left(\theta ; x_{1}, \cdots, x_{n}\right)
$$

Then, log-likelihood function is

$$
\mathbf{L}_{*}\left(a_{1}, b_{1}, \sigma_{e}\right)=\frac{-n}{2} \ln 2 \pi \sigma_{e}^{2}-\frac{\mathbf{S}_{*}\left(a_{1}, b_{1}\right)}{2 \sigma_{e}^{2}}
$$

where, $\mathbf{S}_{*}\left(a_{1}, b_{1}\right)=\sum_{t=1}^{n} e_{t}^{2}\left(a_{1}, b_{1} \mid y_{*}, e_{*}\right)$

Moreover,

$$
\hat{\sigma}_{e}^{2}=\frac{S_{*}\left(\hat{a}_{1}, \hat{b}_{1},\right)}{d . f}
$$

where $d . f=n o$. of terms in $S_{*}-$, no. of parameters.

\section{Particle Swarm Optimization}

\subsection{Description of PSO Algorithm}

Optimize an $d$-dimensional objective function $f(\vec{x})$, the original PSO algorithm is initialize with a population of compute solutions (called particles) $\left\{p_{1}, \cdots, p_{k}\right\}=P$, randomly located in the solution space. The objective function determines the quality of particle's position, the equality of the solution it represent.

A particle $p_{i}$ at time step $g$ has a position vector $\vec{x}_{i}^{g}$ and an associated velocity vector $\vec{v}_{i}^{g}$, every particle remembers the position in which he it has received the best evolution of the objective function. This memory is represented by a vector $p \vec{b} e s t_{i}$, this vector is updated every iteration particle $p_{i}$ finds a better position.

The vector $g \vec{b}$ est stores the best position any particle in the swarm has visited, so for the minimization problem 
$f(g \vec{b} e s t) \leq f(p \vec{b} e s t) \forall p_{i} \in P$, the same. The algorithm updating the particle's velocity and the position iteratively until the stopping criterion valid.

The population of $P S O$ is the swarm consist of a number of particles, a particle in the swarm has inter connected with other particles, this inter connected is called the neighborhood topology, where the neighborhood used for a communication structure not for geographical neighborhood. The particles moving in the search space by following a change rule, based on this rule the particles move through the search space at a given iteration $t$ depending on its position at iteration $t-1$ as well as the position of its previous best location, this describe of phase cognitive of PSO. The social phase of PSO introduces by an interaction rule, where the particles position are not sufficient by the best position in there history, but also on the best position in history of there neighbors.

The particle has three $d$-dimensional principles vectors,

$\vec{x}_{i}$ : Current position.

$\vec{p}_{i}$ : Previous best position.

$\vec{v}_{i}$ : Velocity used to update the position, velocity used as a step size to modified algorithm's solution which is adjusted through the algorithm's computations.

Equations used for updating the velocities and the positions are,

$$
\begin{gathered}
\vec{v}_{i} \leftarrow \vec{v}_{i}+\vec{U}\left(0, \varphi_{1}\right) \otimes\left(\vec{p}_{i}-x_{i}\right) \\
+\vec{U}\left(0, \varphi_{2}\right) \otimes\left(\vec{p}_{g}-\vec{x}_{i}\right) \\
\vec{x}_{i} \leftarrow \vec{x}_{i}+\vec{v}_{i}
\end{gathered}
$$

$\vec{U}\left(0, \varphi_{i}\right)$ : Vector of randoms numbers uniformly distributed in $\left(0, \varphi_{i}\right)$ generated randomly at each iteration and for each particle.

$\otimes:$ Component-wise multiplication, and $\phi_{i}$ : Fixed parameters known as acceleration parameters, they determine the magnitude of the random forces in the direction of best personal position $\vec{p}_{i}$ and neighborhood best $\vec{p}_{g}$.

\subsection{PSO Algorithm Implementation}

The real description of $P S O^{\prime} \mathrm{s}$ mechanism explaining by the following three basic steps:

(1) Initialization: The positions of the particle in the search space they are initialized uniformly random within the search space. If one choose to initialize the velocities to a vector of zero then $\vec{p}_{i}$ should be different from $\vec{x}_{i}$ to enable the particle to start moving, usually $\vec{x}_{i}$ and $\vec{v}_{i}$ are initialized randomly while $\vec{p}_{i}$ is initialized as $\vec{x}_{i}$ for the first iteration.

The nonzero velocities move the particle through the search space in a randomly chosen direction and magnitude. pbest $_{i}$ contains the objective function value of the best position of the particle.

(2) Iteration terms and functional evaluation: The function computation is given in general loop where, for each particle, evaluate the desired optimization fitness function in $d$-dimensional variables.

Compere particle fitness evaluation with its Pbest $_{i}$, if current value vec $P_{i}$ equal to the current location $\vec{x}_{i}$ in d-dimensional space :

Identify the particle in the neighborhood with best success so far and assign its index to the variable $g$. change the velocity and position of particle as Eq. (7) if a criterion is met, exit loop.

(3) Stopping criteria

Stopping criteria is used to terminate the iterative search process some of using stopping criteria

- The algorithm is terminated when maximum numbers of iterations function evaluations has been reached, If the maximum number of iterations is too small the search process may stop before a good results has been found.

- The algorithm is terminated when there is no significant improvement over a number of iterations, this improvement can be measured in different ways. The process may be considered to have terminated if the average change of the particles position are very small or the average velocity of the particles is approximately zero over a number of iterations. 


\subsubsection{Canonical PSO Algorithm}

The scheme of canonical PSO algorithm to the minimization problem given by the following figure.

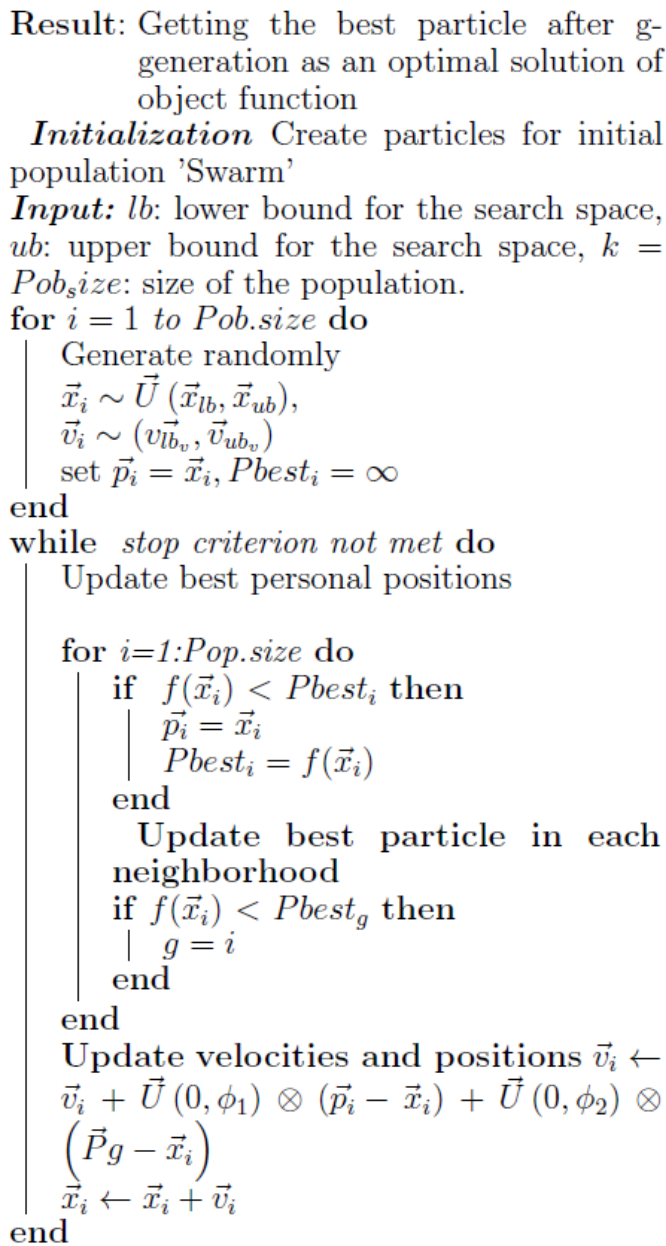

Fig. 2 Schem of canonical PSO algorithm.

\subsection{Neighborhood Topology}

Population typologies work as a method to select the vector $l b \vec{e} t_{i}$ that will guide particle $i$ in its search.

$$
\begin{aligned}
& \forall \text { Par.i,Par.j } \in \text { Swarm : Par. } i \in N(\text { Par. } i) \\
& \Leftrightarrow \operatorname{dist}(\text { Par. } i, \text { Par. } j) \leq \delta
\end{aligned}
$$

$N($ Par. $i)$ is a topological neighbor of particle Par.i

The most popular types of population's neighborhood typologies used in this work are:

Fully connected topology, (Gbest topology) This type of topology the particle's neighborhood contains all particles in the population, where in the evolutionary process, only the particle that obtains the optimal position is considered in the entire population and $P S O$ algorithms with this topology converse very fast but easy to fall into local optimum [4].

Ring topology -( lbest topology) The particle's neighborhood includes the particles on both sides of one or a few particles, the exchange of information is relatively slow within the population, as a result the information eventually will slowly spread to the entire population. The following figure give the method of connections between the population particles in the given neighborhood typologies [4].
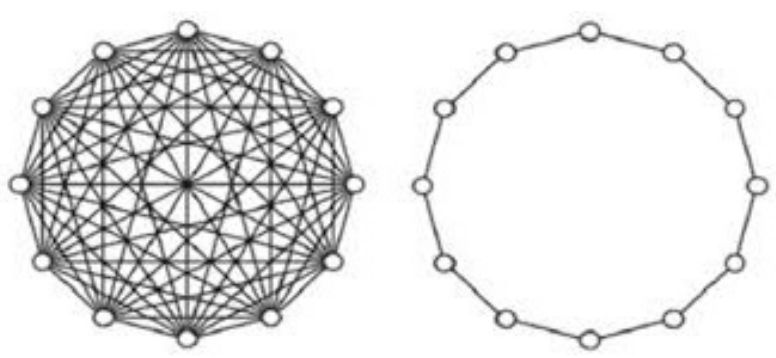

Fig. 3 Gbestand and Lbest population's neighborhood typologies.

\subsection{Versions of Canonical PSO}

There are many of versions of PSO founded, this versions given by updating equation of the velocity, versions used in this study are:

\subsubsection{Constriction Coefficient PSO Algorithm CCPSO}

Clerc and Kennedy, 2002 introduced a constriction factor into the velocity update rule of the original PSO algorithm, the purpose of this factor is to avoid particles velocities to increase toward infinity,a phenomenon present in the original PSO, the updating equations of velocity and positions

$$
\begin{gathered}
\vec{v}_{i} \leftarrow \chi\left(\begin{array}{c}
\vec{v}_{i}+\vec{U}\left(0, \varphi_{1}\right) \otimes\left(\vec{p}_{i}-\vec{x}_{i}\right) \\
+\vec{U}\left(0, \varphi_{2}\right) \otimes\left(\vec{p}_{g}-\vec{x}_{i}\right)
\end{array}\right) \\
\vec{x}_{i} \leftarrow \vec{x}_{i}+\vec{v}_{i} \\
\phi=\phi_{1}+\phi_{2}>4 \text {, and } \chi=\frac{2}{\varphi-2+\sqrt{\varphi^{2}-4 \varphi}}
\end{gathered}
$$




\subsubsection{Inertia Weight PSO Algorithm IWPSO}

In 1998, first time Shi and Eberhart [2] presented the concept of Inertia Weight by introducing Constant Inertia Weight. They stated that a large Inertia Weight facilitates a global search while a small Inertia Weight facilitates a local search.

Eberhart and Shi proposed a Random Inertia Weight strategy and experimentally found that this strategy increases the convergence of PSO in early iterations of the algorithm. The Linearly Decreasing strategy enhances the efficiency and performance of PSO. It is found experimentally that Inertia Weight from 0.9 to 0.4 provides the excellent results, the updating equations of this version are:

$$
\begin{gathered}
\vec{v}_{i} \leftarrow w \vec{v}_{i}+\vec{U}\left(0, \varphi_{1}\right)\left(\vec{p}_{i}-\vec{x}_{i}\right) \\
+\vec{U}\left(0, \varphi_{2}\right) \otimes\left(\vec{p}_{g}-\vec{x}_{i}\right) \\
\vec{x}_{i} \leftarrow \vec{x}_{i}+\vec{v}_{i} \\
w=w_{\max }-\left(w_{\max }-w_{\min }\right) \frac{g}{G}
\end{gathered}
$$

$g$ is the generation index representing the current number of evolutionary.

$G$ is a predefined maximum number of generations.

\subsection{Fully Informed Particle Swarm 'FIPSO'}

Fully Informed Particle Swarm Optimization 'FIPSO', is a variant of PSO algorithm found by Kennedy and Mendes 2002 [5], in canonical version of PSO, the effective sources of influence are in fact only two: self and best neighbor, information from the remaining neighbors is unused. Mends has revised the way particles interact with there neighbors, whereas in the traditional algorithm each particle is effected by its own previous performance and the single best success found in its neighborhood, FIPSO calls for the particles to take advantage of the connections with others, his neighbors in the swarm. The implementation of FIPS with good parameters appears to find better solutions in fewer iterations than the canonical algorithm, but it is still more dependent on the population topology.

$$
\begin{aligned}
& \vec{v}_{i} \leftarrow \chi\left(\vec{v}_{i}+\frac{1}{N_{i}} \sum_{n=1}^{N_{i}} \vec{U}(0, \varphi) \otimes\left(\vec{p}_{n g h}-\vec{x}_{i}\right)\right) \text { (10) } \\
& \vec{x}_{i} \leftarrow \vec{x}_{i}+\vec{v}_{i} \\
& N_{i} \text { is the number of neighbors for particle i, } \\
& n g h_{n} \text { is i's nth neighbor. }
\end{aligned}
$$

Input: $l b$ : lower bound for the search space, $u b$ : upper bound for the search space, $k=$ $\mathrm{Pob}_{s} i z e$ : size of the population.

Initialization Create particles for initial population 'Swarm' for $i=1$ to Pob.size do

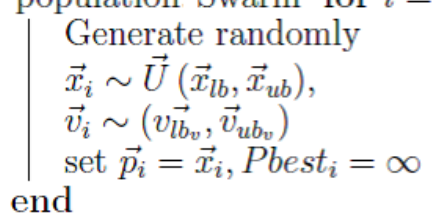

while stop criterion not met do

Update best personal positions

for $i=1$ :Pop.size do

$$
\begin{aligned}
& \text { if } f\left(\vec{x}_{i}\right)<\text { Pbest }_{i} \text { then } \\
& \mid \begin{array}{l}
\vec{p}_{i}=\vec{x}_{i} \\
\text { Pbest }
\end{array}=f\left(\vec{x}_{i}\right) \\
& \text { end }
\end{aligned}
$$

Update best particle in each neighborhood

if $f\left(\vec{x}_{i}\right)<$ Pbest $_{g}$ then

$g=i$

end

end

Update velocities and positions

$\vec{v}_{i}=\chi\left(\vec{v}_{i}+\sum_{n=1}^{N_{i}} \frac{\vec{U}(0, \phi) \otimes\left(\vec{p}_{n g h(n)}-\vec{x}_{i}\right)}{N_{i}}\right)$,

$\vec{x}=\vec{x}_{i}+\vec{v}_{i}$

end

Fig. 4 Schem of FIPSO algorithm.

\section{Experimental Section}

The data generated by $\operatorname{ARMA}(1,1)$, for $e_{t \sim} N(0,1)$

$$
y_{t}=a_{1} y_{t-1}+e_{t}-b_{1} e_{t-1}
$$

The numerical computation of conditional log-likelihood function given by the following steps 
For sample size equal to $n$, and given $a_{1}, b_{1}$ generate time series measurements $y_{t}, t=1, \cdots, n$ as Eq. (4), where $e_{t}, t=, \cdots, n$ generated randomly as $N(0,1)$.

Set initial value of $e_{*}=0$, and $y_{*}=\bar{y}$, sample mean.

Let $(a, b)$ be a selected candidate solutions in the search space, then for $t=1, \cdots, n$ calculate $e$ 's recursively as Eq. (4)

$$
e_{t}=y_{t}-\hat{a}_{1} y_{t-1}+\hat{b}_{1} e_{t-1}
$$

Compute the sum squares function for every $\left(\hat{a}_{1}, \hat{b}_{1}\right)$.

Compute log-likelihood function.

Set $\left(\hat{a}_{1}, \hat{b}_{1}\right)$ to be the optimal solution of Eq. (6) values of model parameters corresponding to a maximum value of log-likelihood function.

Remark Conditional likelihood $l_{*}$ involves the data only through the conditional sum of squares function. It follows that the contours of $l_{*}$ for any fixed value of $\sigma_{e}$ in the space of $\left(a_{1}, b_{1}, \sigma_{e}\right)$ are counters of $S_{*}$, that maximum likelihood estimators are the same as the least squares estimates.

\section{Experiments setting}

Experiments designed by different setting of Pop.size, initial velocity, type of terminate criterion, type of neighborhood topology, sample size, model parameters,

Setting $\operatorname{ARMA}(1,1)$ parameters: The experiments simulated by using different values of objective function parameters

Sample size, $n=25,50,100$.

Model Parameters $\left(a_{1}, b_{1}\right),(0.1,-0.2),(0.3,0.2)$,

\section{$(.7, .3)$.}

Setting PSO algorithm parameters: The basic PSO algorithm has a small number of parameters that need to be fixed:

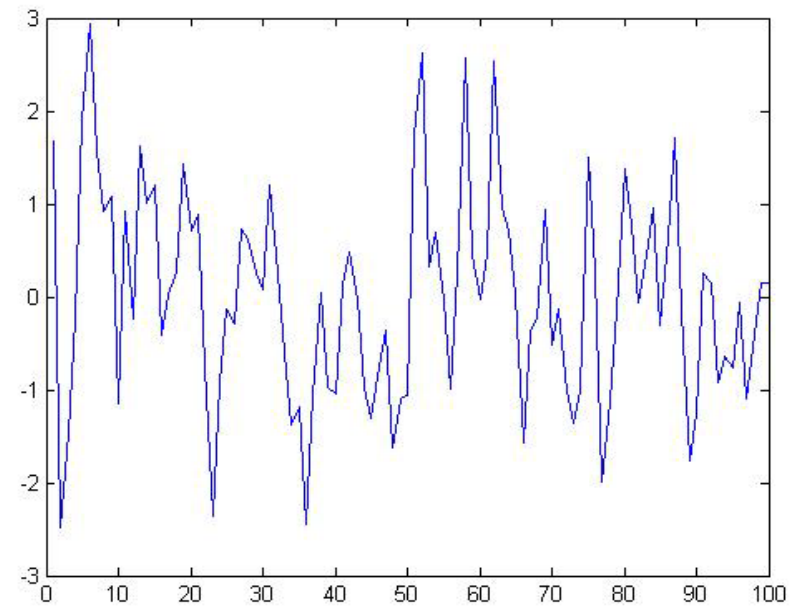

Fig. 5 Simulating data generated by $\operatorname{ARMA}(1,1)$.

Pop.size : Population size is often set empirically on the basis of the dimensional and perceived difficulty of the problem, best range of population size is 20-50, here Pop.size $=20$.

Acceleration coefficients: setting $\left(\phi_{1}=\phi_{2}=2.05\right)$.

In general: The initial population generated randomly for $a_{1}, b_{1}$ in $(-1,1)$.

Initial velocity generated randomly in the same range of the positions or set it equal to zero may be not the right choose, the results show putting big values of velocities leads to search out of problem search space, for this study the velocities take the initial generated randomly for $i=1$ to Pop.size in the range $(-0.08, .08)$, and number of iterations set equal to 100 .

The test statistic used Mean Squares Errors MSE

$$
\operatorname{MSE}(\theta)=\operatorname{var}(\hat{\theta})+\operatorname{bias}(\theta)^{2}
$$

$M S E$ computed for the best estimator found by PSO's algorithms, each experiment replicated for 10 times.

The results given a table. 1 and Fig. 6 for measuring $M S E$ of estimator getting by versions of $P S O$ 's algorithm respect to sample size, Fig. 7, Fig. 8 show the value of obj.fun respect to $P S O$ 's iterations for one run and mean of 10 runs. We can see from the values of MSE the ability of $P S O$ 's algorithms to estimate $A R M A$ 's parameters, the values of $M S E$ decreasing with increasing sample size, COPSO algorithm gives 
the smallest values of MSE. Smallest MSE's values found for $\left(a_{1}, b_{1}\right)=(.7,3)$ and $n=100$. CCPSO and IWPSO used to foster the convergence ability by controlling the velocities, FIPSO used to share information more quickly in the swarm in order to achieve the optimal solution in fewer iterations, comparing the results of this study with results given in [8], [9] and [10], show that PSO is easy to implement, and efficient for computing the optimization solution with few iteration where after few iterations the values of obj.fun being stable, results of this study will get better by adding the steps of mutation and crossover to the computing steps this will be in the future study.

We can use this estimators to show the prediction of time series data. After applying versions of PSO algorithm to estimate parameters, a simple experiment designed to predict observations generated by
$\operatorname{ARMA}(1,1)$ time series model for sample size $n=100$, predict value of the observations given in Fig. 9 respect to time $t$ for different versions of $P S O$ algorithm using in this study.

\section{Conclusion}

In general, PSO is an intelligent algorithm has no overlapping and mutation calculation, easy to implement and compute. The results show ability of $P S O$ 's algorithms to estimate ARMA parameters. For the future work, extending this study to cover estimation of like $\operatorname{ARMA}(p, q), G R A C H$ models, and others models, using $P S O$ as tool to model selections and prediction, improving results of this study by hybridizing PSO algorithm with gradient algorithms or other soft computing algorithms and applying the results on a real data like financial data, industrial data, $\cdots$.

Table 1 MSE of parameters estimation for different sample size and PSO versions for model parameters estimation.

\begin{tabular}{|c|c|c|c|c|c|c|c|}
\hline \multirow{2}{*}{$n$} & \multirow{2}{*}{$a_{1}, b_{1}$} & \multicolumn{2}{|c|}{ CCPSO } & \multicolumn{2}{|c|}{ IWPSO } & \multicolumn{2}{|c|}{ FIPSO } \\
\hline & & $\overline{\left(a_{1}\right)}$ & $\left(b_{1}\right)$ & $\left(a_{1}\right)$ & $\left(b_{1}\right)$ & $\left(a_{1}\right)$ & $\left(b_{1}\right)$ \\
\hline 25 & & .3052 & .3804 & .4458 & .7585 & .3425 & .4637 \\
\hline 50 & $.1,-.2$ & .1249 & .2159 & .1820 & 0.356 & 1.1578 & .4191 \\
\hline 100 & & .1040 & .093 & .1171 & .1205 & .1056 & .2705 \\
\hline 25 & & .1086 & .4894 & .1562 & .4956 & .1295 & .4224 \\
\hline 50 & $.3, .23$ & .0318 & .4063 & .0724 & .4850 & .972 & .3769 \\
\hline 100 & & .0182 & .3059 & .0194 & .3392 & .0315 & .3000 \\
\hline 25 & & .0891 & .6581 & 0.060 & 8.4925 & .0585 & .4910 \\
\hline 50 & $.7, .3$ & .0083 & .4842 & .0117 & .4902 & .0092 & .4759 \\
\hline 100 & & .0028 & .4556 & .0029 & .4678 & .0027 & .4651 \\
\hline
\end{tabular}
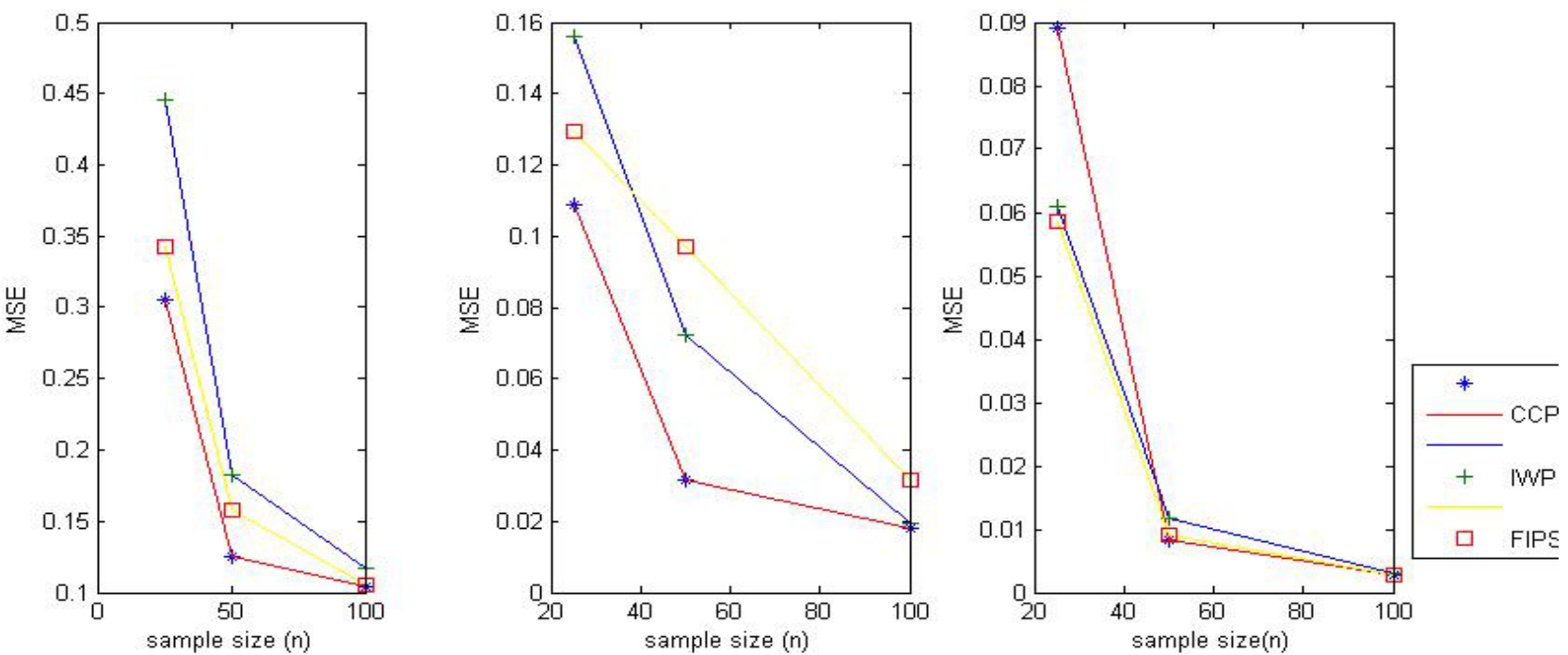

Fig. 6 MSE respect to sample size(n) for COPSO, IWPSO, FIPSO. 
Simulating Particle Swarm Optimization Algorithm to Estimate Likelihood Function of $A R M A(1,1)$ Model 407
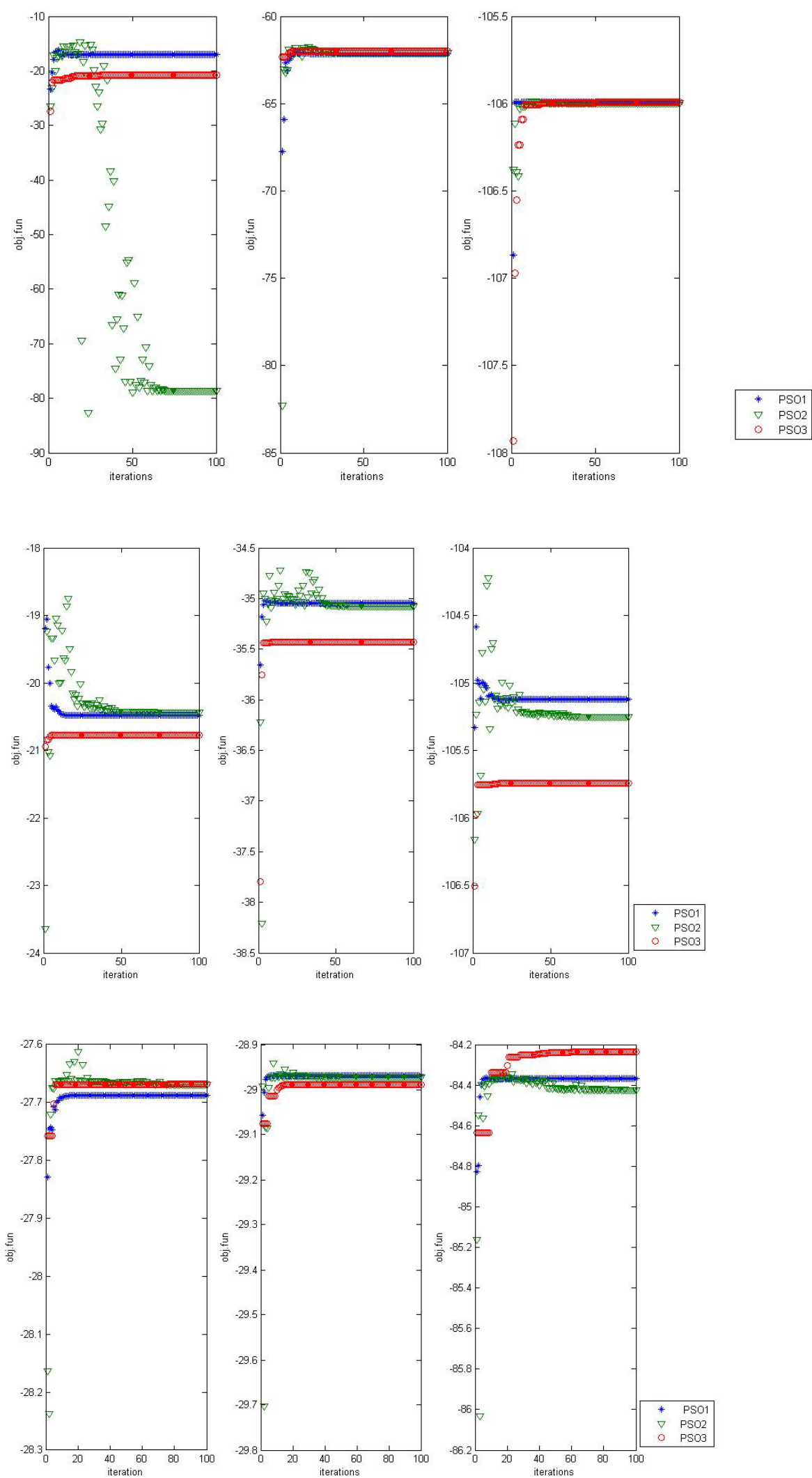

Fig. 7 Average of Obj.fun respect to number of generations for different sample size(n), PSO' versions (COPSO, IWPSO and FIPSO respectively), for 10 replicates 

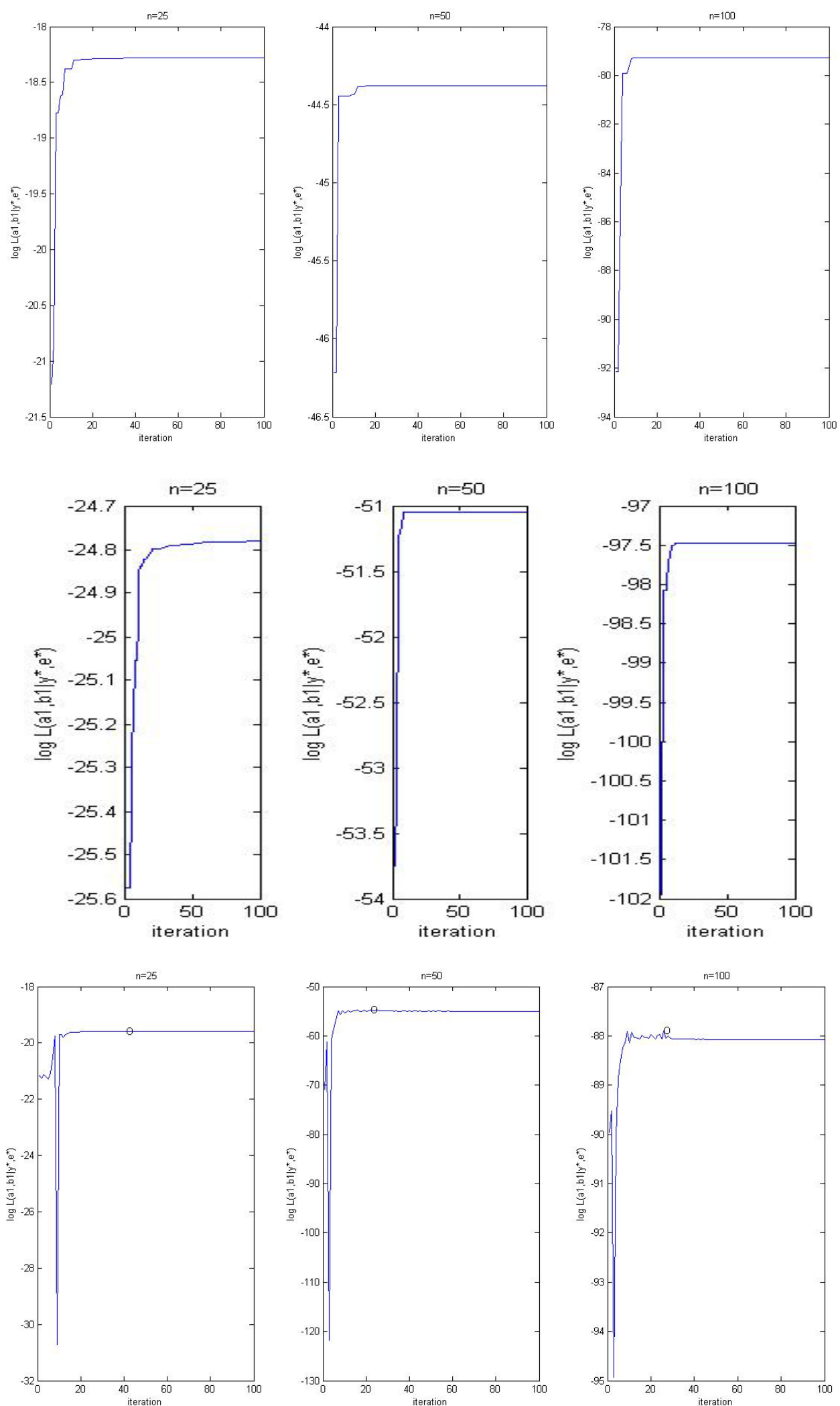

Fig. 8 Obj.fun respect to number of generations for different sample size(n), PSO' versions (COPSO, IWPSO and FIPSO respectively), and 1 replicate 

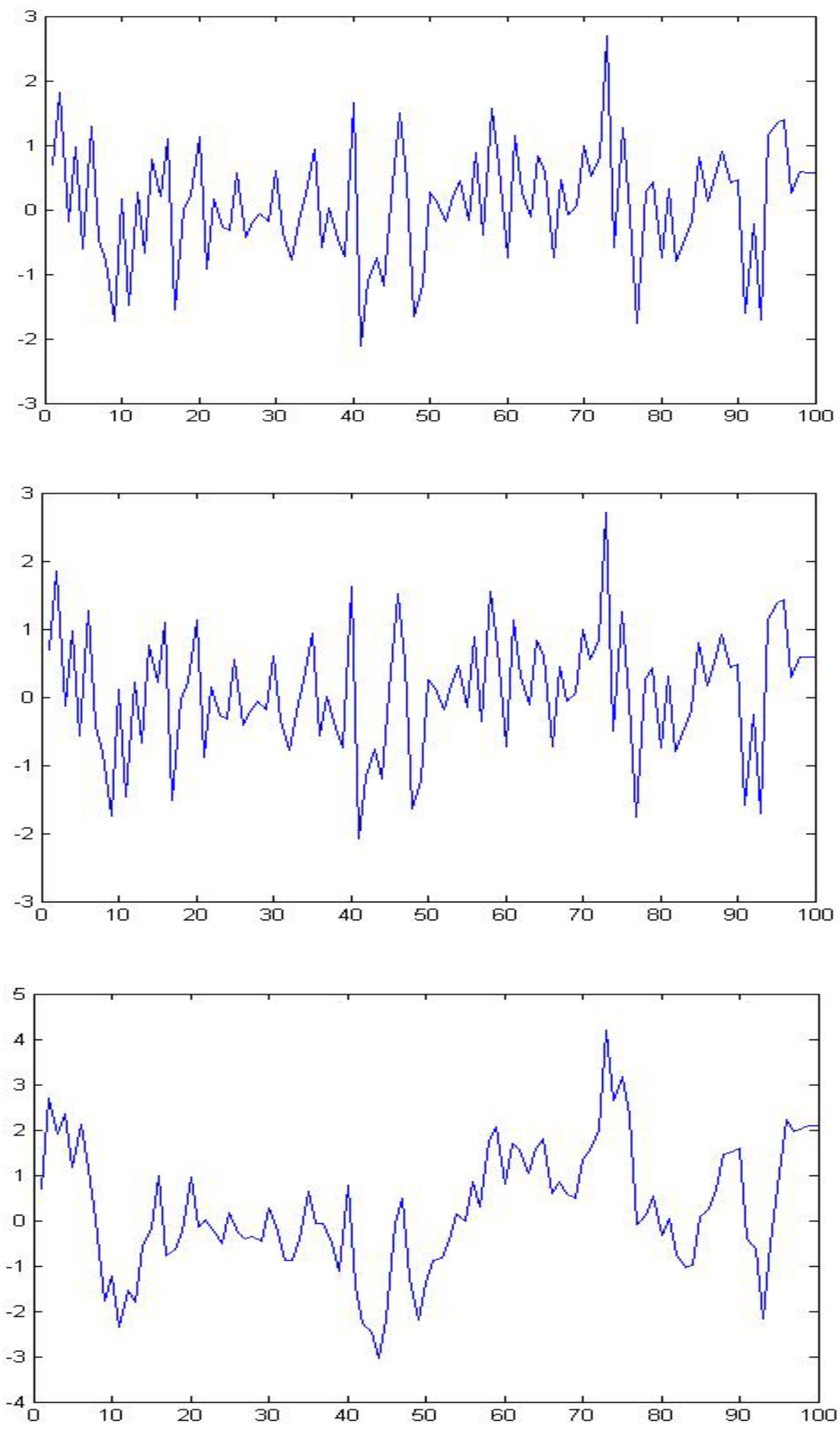

Fig. 9 Prediction of generated observation $y_{t}$ based on $P S O$ 's estimators, where figuers given respectively for $C C P S O$, IFPSO, FIPSO, for $a_{1}=: 7 ; b_{1}=: 3$ and $n=100$ 


\section{Acknowledgment}

The author thank LMB, Universite de Franche-Comte, CNRS - UMR 6623 \& Ministry of higher education in Iraq (Baghdad university), for their support and for financing this project.

\section{References}

[1] R.Eberhart\& J. Kennedy. (1995). A New optimizer using particle swarm theory, IEEE-Sixth international Symposium on Micro Machine and Human Science, 19-27.

[2] D.P.Rini \& S.M.Shamsuddin \& S.S.Yuhaniz. (2011). Particle swarm optimization: techique, system and challenges. International journal of computer applications 14, 331-351.

[3] Z.Zhan \& J.Zhang \& Y.Li, H.S.Chung. (2009). Adaptive Particle swarm optimization, IEEE-Transactions on system, man, and Cyberntic-part B 39, 1362-1381

[4] A.J.R. Medina \& G.T. Pulido \& J.G.R. Torres. (2009). A comparative study of neighborhood typologies for particle swarm optimizer, IJCCI2009, 152-159.

[5] J.Kennedy \& R. Mendes. (2006), Neighborhood topologies in fully informed and best-of-neighborhood particle swarms. IEEE, Vol.36,Issue.4, 515-519.

[6] W.W.S.Wei. (1994). Time series analysis, Univariate and multivariate methods, Addison-Wesley publishing company,Inc.

[7] G.E.B.Box \& G.M. Jenkins. (1976). Time series analysis: Forecasting and control, Holden-Day, San Francisco

[8] B.A.h. Al-sarray \& R.D.Al-dabbagh. (2011). Variants of hybrid genetic algorithms for optimizing likelihood ARMA model function and many of problems, InTechOpen, Published on: 2011-04-26.

[9] B.A.h. Al-sarray \& R.D. Al-dabbagh. (2008). Hybrid canonical genetic algorithm and steepest descent algorithm for optimizing likelihood estimators of $\operatorname{ARMA}(1,1)$ model. ICADIWT2008, Ostrava, Czech Republic.

[10] B.A. Attea \& B.A.h. Al-sarray. (2006). An Evolution Strategy for Likelihood Estimators of ARMA(1,1) Model. CSIT2006, Amman, Jordan. 